\title{
Methanol masers survey using EVN
}

\author{
Anna Bartkiewicz* \\ Centre for Astronomy, Nicolaus Copernicus University \\ Gagarina 11, 87-100 Torun, Poland \\ E-mail: annaneastro.uni.torun.pl \\ Marian Szymczak \\ Centre for Astronomy, Nicolaus Copernicus University \\ Gagarina 11, 87-100 Torun, Poland \\ E-mail: msz@astro.uni.torun.pl
}

\section{Huib Jan van Langevelde}

Joint Institute for VLBI in Europe,

Postbus 2, 7990 AA Dwingeloo, The Netherlands

Sterrewacht Leiden, Postbus 9513, 2300 RA Leiden, The Netherlands

E-mail: langevelde@jive.nl

We present the results of five year campaign observing $6.7 \mathrm{GHz}$ methanol masers towards the Galactic plane using European Very Long Baseline Interferometer Network (EVN). 31 out of 33 sources were imaged at milliarcsecond scale. Suprisingly, 12 of them showed an elliptical morphology which has not been detected before. We state that the recent increase of the sensitivity of EVN allowed us to detect a new type of masers. We discuss the origin of elliptically shaped methanol masers in massive star forming regions analysing their detailed structures.

The 9th European VLBI Network Symposium on The role of VLBI in the Golden Age for Radio Astronomy and EVN Users Meeting

September 23-26, 2008

Bologna, Italy

${ }^{*}$ Speaker. 


\section{Introducion}

Methanol maser emission at $6.7 \mathrm{GHz}$ is well know due to its strong association with highmass star-forming regions [5]. Due to its brightness and compactness the maser is widely used in investigation of close (100-1000 AU) surroundings of protostars. VLBI observations with milliarcsecond (mas) scale have been carried out for many years to date. The structures of the maser emission are from simple to complex structures indicating they may form in outflows or behind shocks driven by a protostar or in discs/torii around a central object ([4], [6], [8], [9], [13]). Their origin has not been established yet.

Here we present the summary of our project to observe methanol maser sources carried out in 2003-07 using the EVN ${ }^{1}$ The recently increased sensitivity of EVN enabled us to detect weak features of the maser and consequently to find a new morphology not observed so far. The new maps allowed us to investigate the scenarios of methanol maser emission in great details.

A full description of the project and disscussion are being prepared for publication in Astronomy \& Astrophysics and the parts of the result were presented at the previous EVN Symposia ([1]; [2]).

\section{Observations and data reduction}

31 sources with $6668.519 \mathrm{MHz}$ methanol maser emission were selected for VLBI observations. They all were discovered using the Torun $32 \mathrm{~m}$ antenna ([10], [11]) and their positions were improved with astrometric measurements of MERLIN (Cm-Mk II). In addition, two sources known from the literature ([13], [3]) that are located in the same region on the sky were included. The EVN observations towards all 33 targets were carried out on seven runs in June 2003 (4 antennas), November 2004 (8 antennas), February 2006 (7 or 8 antennas) and June 2007 (8 or 9 antennas). Typically five or six sources were observed in each run. Phase-reference sources were chosen carefully for each group of maser from the VLBA calibrator list. Typically, a total onsource time for the target was about $41 \mathrm{~min}$. The bandwidth was $2 \mathrm{MHz}$ in both circular hands. The data were correlated with 1024 spectral points yielding a velocity resolution of $0.09 \mathrm{~km} \mathrm{~s}^{-1}$. In the case of nine antenna observations the correlation was with 512 points due to the limit of correlator (MK IV Data Processor operated by JIVE).

The data calibration and reduction were carried out with the standard procedures for spectral line observations using the Astronomical Image Processing System (AIPS). The absolute position of the target, owing to the phase-referencing, is estimated to be 12 mas in Dec and 10 mas in RA. In the final step the targets were self-calibrated on a strong and point-like maser spot identified for each target. The analysis was carried out on images $0.5 \times 0.5 \operatorname{arcsec}^{2}$ obtained with natural weighting, a typical beam was 6 mas $\times 16$ mas elongated along NS. The rms noise level $(1 \sigma)$ in line-free channels was $3-12$ mJy beam ${ }^{-1}$ depending on the run.

\footnotetext{
${ }^{1}$ The European VLBI Network is a joint facility of European, Chinese, South African and other astronomy institutes funded by their national research councils.
} 


\section{Results}

We obtained images for 31 out of 33 targets and were able to apply the phase-referencing scheme in 29 sources deriving the absolute positions. In total almost 2000 maser spots were detected above $5 \sigma$ level. When a spot appeared at least three consequtive channels we checked its profile. $80 \%$ of all profiles (265 out 333) showed Gaussian characteristic with the mean FWHM of $0.40 \pm 0.01 \mathrm{~km} \mathrm{~s}^{-1}$.

We classify the $6.7 \mathrm{GHz}$ methanol maser emission into six morphological types: simple (1), linear (5), elliptical (12), arched (3), complex (9) and pair (1). The number of sources of each type are given in the brackets. That classification is simply based on the distribution of spots on the sky plane. In Fig. 1 we present examples of sources representing each class and in Table 1 we summarize the results of our project. The main parameters are listed for each target: the coordinates of the brightest spot, the range of LSR velocity of the emission, the peak of the flux density, the number of registered spots and the morphology.

\section{Discussion}

The most unexpected result in the survey is a large number of elliptical shaped methanol maser sources. We suppose that superb sensitivity of EVN enabled us to detect such structures, in relatively weaker targets $\left(S_{p}=\leq 28.3 J y\right)$. A typical peak flux density in the cross-correlated spectra is $3.6 \mathrm{Jy} \mathrm{beam}^{-1}$, while in the previous surveys it was 10-100 times higher ([8]; [9]; [6]; [13]; [4]).

The elliptical morphology strongly supports the scenario of an inclined disc or torus around a massive protostar or young star. A model of a rotating and expanding maser ring has been recently proposed [12]. We applied that model to 12 sources from the sample that have elliptical morphology. In general, the best fits obtained suggest that the expansion velocity dominates over the rotation component in a majority of the sources. It suggests that the maser arises in the zone where the rotation still exists but the expansion plays a role. That can take place between the rotating disc/torus and the outflow.

A similar case towards the well-known object Cep A has been reported during this Symposium (Sugiyama et al. and Torstensoon et al. this volume). They found that the elliptically distributed 6.7 GHz methanol masers were detected around the H II region HW 2. The plane of the ellipse is perpendicular to the bipolar outflow. The LSR velocity distributions of maser spots show similar characteristic that the expansion dominates over the rotation. In order to verify if similar scenario is going on in the targets from the sample we need infrared observations. However, the available angular resolution in the infrared wavelenght range is still limited. The proper motion VLBI studies may be a crucial for these studies.

\section{Conclusions}

We completed the $6.7 \mathrm{GHz}$ methanol line survey in the Galactic plane for a sample of 33 maser sources. High quality EVN images were taken for 31 targets. In most cases the masers show complex structures. The observed morphologies can be divided into six groups. It is suprising 
Table 1: Results of EVN observations taken at epochs 2003-07

\begin{tabular}{|c|c|c|c|c|c|c|}
\hline \multirow{2}{*}{$\begin{array}{l}\text { Source } \\
\text { Gll.1ll } \pm \text { bb.bbb }\end{array}$} & \multicolumn{2}{|c|}{ " Accurate position (J2000) } & \multirow{2}{*}{$\begin{array}{c}\mathrm{V}_{\mathrm{s}} ; \mathrm{V}_{\mathrm{e}} \\
\left(\mathrm{km} \mathrm{s}^{-1}\right)\end{array}$} & \multirow{2}{*}{$\begin{array}{c}\mathrm{S}_{\mathrm{p}} \\
\left(\mathrm{Jy} \mathrm{beam}^{-1}\right)\end{array}$} & \multirow{2}{*}{$\begin{array}{c}\text { Number } \\
\text { of } \\
\text { spots }\end{array}$} & \multirow[t]{2}{*}{ Morph } \\
\hline & $\begin{array}{l}\text { RA } \\
(\mathrm{h} \mathrm{m} \mathrm{s})\end{array}$ & $\begin{array}{l}\text { Dec } \\
\left({ }^{\circ}\right), ",\end{array}$ & & & & \\
\hline G21.407-00.254 & 183106.33794 & -102137.4108 & $88.7 ; 91.7$ & 2.76 & 26 & $\mathrm{C}$ \\
\hline G22.335-00.155 & 183229.40704 & -092929.6840 & $35.3 ; 38.4$ & 1.71 & 12 & $\mathrm{~L}$ \\
\hline $\mathrm{G} 22.357+00.066$ & 183144.12055 & -092212.3129 & $79.5 ; 88.7$ & 10.54 & 31 & $\mathrm{C}$ \\
\hline G23.207-00.377 & 183455.21212 & -084911.8926 & $72.3 ; 85.5$ & 9.30 & 190 & $\mathrm{E}$ \\
\hline $\mathrm{G} 23.389+00.185$ & 183314.32477 & -082357.4723 & $71.8 ; 77.8$ & 21.55 & 128 & E \\
\hline G23.657-00.127 & 183451.56482 & -081821.3045 & $77.0 ; 87.8$ & 3.62 & 315 & $\mathrm{E}$ \\
\hline G23.707-00.198 & 183512.36600 & -081739.3577 & $58.2 ; 81.5$ & 6.06 & 140 & A \\
\hline G23.966-00.109 & 183522.21469 & -080122.4698 & $67.2 ; 71.4$ & 5.47 & 25 & $\mathrm{~L}$ \\
\hline G24.147-00.010 & 183520.92949 & -074900.1800 & $17.0 ; 18.6$ & 5.20 & 22 & $\mathrm{~L}$ \\
\hline $\mathrm{G} 24.541+00.312$ & 183455.72152 & -071906.6504 & $103.6 ; 110.4$ & 7.75 & 73 & A \\
\hline G24.634-00.324 & 183722.71271 & -073142.1439 & $34.7 ; 48.1$ & 3.03 & 23 & $\mathrm{E}$ \\
\hline $\mathrm{G} 25.411+00.105$ & 183716.92106 & -063830.5017 & $93.7 ; 98.9$ & 3.43 & 30 & $\mathrm{E}$ \\
\hline G26.598-00.024 & 183955.92567 & -053844.6424 & $22.8 ; 26.1$ & 3.04 & 21 & $\mathrm{E}$ \\
\hline $\mathrm{G} 27.221+00.136$ & 184030.54608 & -050105.3947 & $105.2 ; 121.3$ & 12.54 & 173 & $\mathrm{C}$ \\
\hline $\mathrm{G} 28.817+00.365$ & 184237.34797 & -032940.9216 & $87.6 ; 92.8$ & 3.14 & 28 & $\mathrm{E}$ \\
\hline G30.318+00.070 & 184625.02621 & -021740.7539 & $35.2 ; 37.1$ & 0.52 & 8 & $\mathrm{~L}$ \\
\hline G30.400-00.296 & 184752.29976 & -022316.0539 & $97.9 ; 104.6$ & 2.77 & 27 & $\mathrm{E}$ \\
\hline G31.047+00.356 & 184643.85506 & -013054.1551 & $77.9 ; 84.2$ & 1.99 & 27 & $\mathrm{E}$ \\
\hline G31.581+00.077 & 184841.94108 & -011002.5281 & $95.1 ; 99.9$ & 2.72 & 28 & $\mathrm{E}$ \\
\hline G32.992+00.034 & 185125.58288 & +000408.3330 & $89.6 ; 94.8$ & 6.21 & 60 & $\mathrm{C}$ \\
\hline G33.648-00.224* & 185332.551 & +003206.525 & $58.4 ; 63.7$ & 28.3 & 94 & A \\
\hline G33.980-00.019 & 185325.01833 & +00 5525.9760 & $58.6 ; 65.5$ & 3.78 & 59 & $\mathrm{E}$ \\
\hline G34.751-00.093 & 185505.22296 & +013436.2612 & $50.4 ; 53.5$ & 1.95 & 30 & $\mathrm{E}$ \\
\hline G35.791-00.175* & 185716.911 & +022752.900 & $59.9 ; 62.7$ & 9.70 & 33 & $\mathrm{~L}$ \\
\hline G36.115+00.552 & 185516.79345 & +030505.4140 & $69.7 ; 84.5$ & 11.74 & 169 & $\mathrm{P}$ \\
\hline G36.705+00.096 & 185759.12288 & +032406.1124 & $52.4 ; 63.0$ & 7.58 & 49 & $\mathrm{C}$ \\
\hline G37.030-00.039 & 185903.64233 & +033745.0861 & $78.3 ; 79.0$ & 0.69 & 8 & S \\
\hline G37.598+00.425 & 185826.79772 & +042045.4570 & $82.8 ; 87.3$ & 3.91 & 31 & $\mathrm{C}$ \\
\hline G38.038-00.300 & 190150.46947 & +042418.9559 & $55.4 ; 59.6$ & 2.17 & 26 & $\mathrm{C}$ \\
\hline G38.203-00.067 & 190118.73235 & +043934.2938 & $78.3 ; 84.3$ & 0.83 & 18 & $\mathrm{C}$ \\
\hline G39.100+00.491 & 190058.04036 & +054243.9214 & $14.5 ; 17.8$ & 2.07 & 31 & $\mathrm{C}$ \\
\hline
\end{tabular}

\footnotetext{
* - coordinates estimated using single baseline of MERLIN (Cm-Mk II)
} 
that about $40 \%$ of the sources exhibit a ring-like spatial distribution of maser spots. This class of methanol sources appears to be consistent with a model of circumstellar disc or torus while their kinematics indicates outflow phenomena.

\section{Acknowledgements}

This work was supported by the MNiI grant 1P03D02729 and UMK grant 407-A.

\section{References}

[1] A. Bartkiewicz, M. Szymczak \& H.J. van Langevelde, Methanol masers in environments of three massive protostars, Proceedings of the 7th European VLBI Network Symposium held in Toledo, eds. R. Bachiller, F. Colomer, J.-F. Desmurs, P. de Vicente, 2004, 187-190

[2] A. Bartkiewicz, M. Szymczak \& H.J. van Langevelde, EVN imaging of Methanol masers towards massive protostars, Proceedings of the 8th European VLBI Network Symposium held in Torun, ed. A. Marecki, 2006, 274-278

[3] H. Beuther, A. Walsh, T.K. Sridharan, K.M. Menten \& F. Wyrowsky, $\mathrm{CH}_{3} \mathrm{OH}$ and $\mathrm{H}_{2} \mathrm{O}_{\text {masers in }}$ high-mass star-forming regions, A\&A, 2002, 390, 289-198

[4] R. Dodson, R. Ojha \& S.P. Ellingsen, High-resolution observations of 6.7-GHz methanol masers with the Long Baseline Array, MNRAS, 2004, 351, 779-790

[5] K.M. Menten, The discovery of new, very strong and widespread interstellar methanol maser transition, ApJ, 1991, 380, L75-78

[6] V. Minier, R.S. Booth \& J.E. Conway, VLBI observations of 6.7 and $12.2 \mathrm{GHz}$ methanol masers toward high mass star-forming regions, A\&A, 2000, 362, 1093-1108

[7] L. Moscadelli, R. Cesaroni \& M.J. Rioja, Tracing the root of the bipolar jet in IRAS 20126+4104: VLBA observations of $\mathrm{H}_{2} \mathrm{O}$ masers, A\&A, 2000, 360, 663-670

[8] R.P. Norris, S.E. Byleveld, P.J. Diamond et al., Methanol masers as traces of circumstellar disks, ApJ, 1998, 508, 275-285

[9] C.J. Phillips, R.P. Norris, S.P. Ellingsen \& P.M. McCulloch, Methanol masers and their environment at high-resolution, MNRAS, 1998, 300, 1131-1157

[10] M. Szymczak, A.J. Kus, G. Hrynek, A. Kepa \& E. Pazderski, A survey of the 6.7 GHz methanol maser emission from IRAS sources. I. Data, A\&AS, 2000, 143, 269-301

[11] M. Szymczak, A.J. Kus, G. Hrynek, A. Kepa \& E. Pazderski, 6.7 GHz methanol masers at sites of star formation. A blind survey of the Galactic plane between $20^{\circ} \leq 40^{\circ}$ and $|b| \leq 0 .{ }^{\circ} 52$, A\&A, 2002, 392, 277-286

[12] L. Uscanga, Y. Gómez, A.C. Raga, J. Cantó, G. Anglada, J.F. Gómez, J.M. Torrelles \& L.F. Miranda Kinematics of the $\mathrm{H}_{2} \mathrm{O}$ masers at the centre of the planetary nebula K3-35, MNRAS, 2008, 390, 1127-1132

[13] A.J. Walsh, M.G. Burton, A.R. Hyland \& G. Robinson, Studies of ultracompact H II regions, MNRAS, 1998, 301, 640-698 
G37.030-00.039 simple

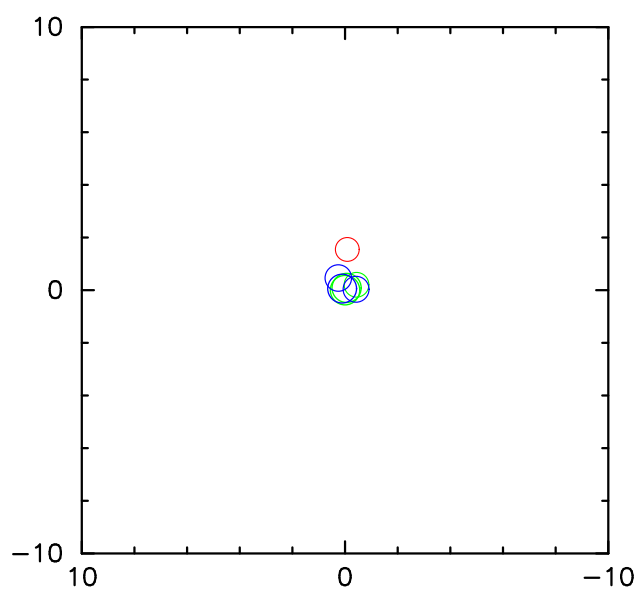

G33.648-00.224 arched

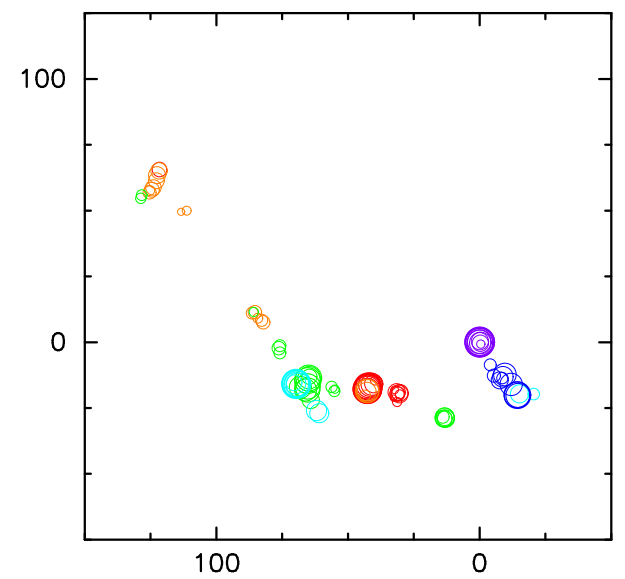

G32.992+00.034 complex

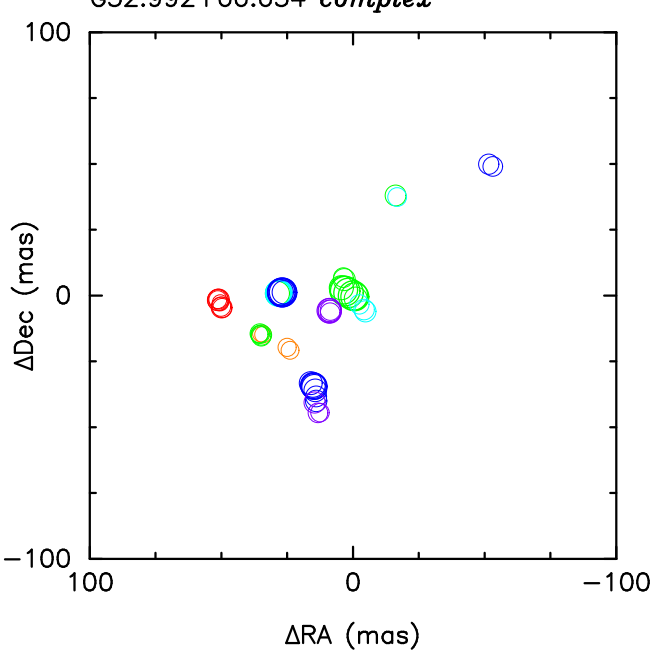

G22.335-00.155 linear

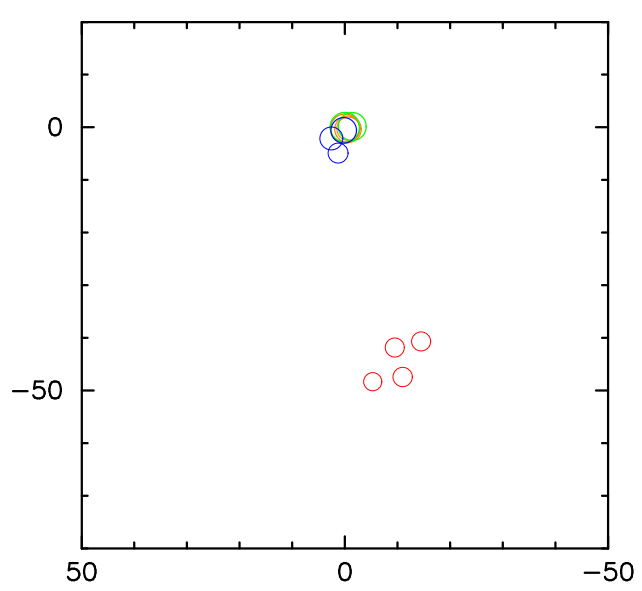

G33.980-00.019 elliptical

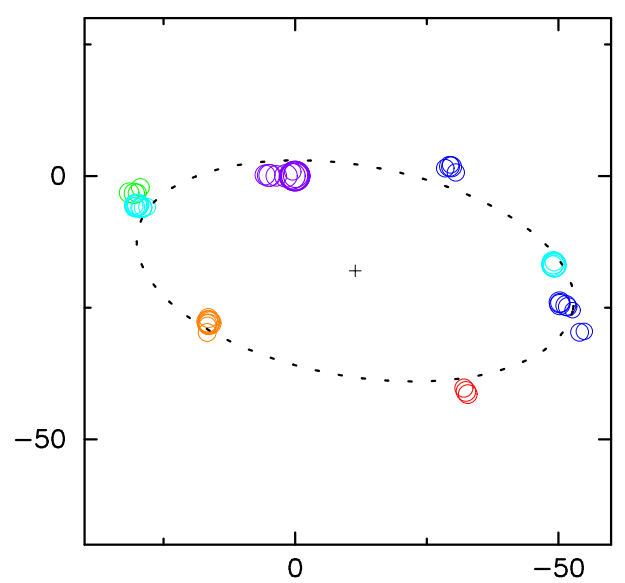

G36.115+00.552 pair

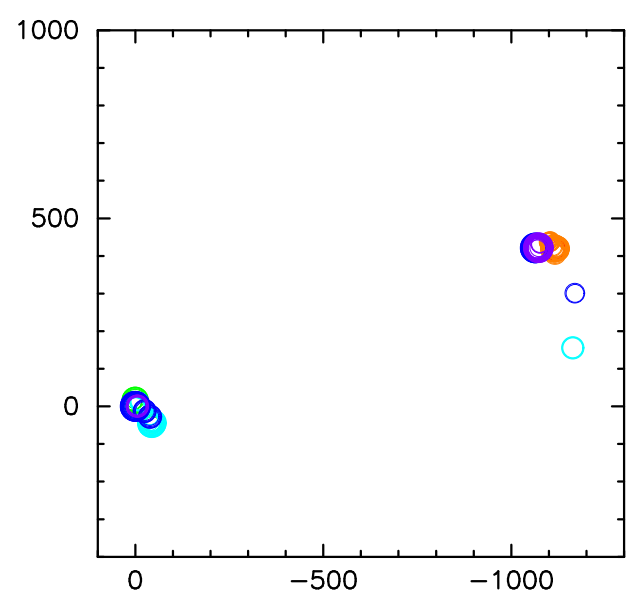

Figure 1: Examples of distribution of $6.7 \mathrm{GHz}$ methanol maser emission. The coordinates are relative to the brightest spots (Table 1). The sizes of circles are proportional to the logarithm of the flux densities. The dashed line traces the best fitted ellipse to the spot distribution in G33.980-00.019. 